\title{
Impact of Pre-eclampsia/eclampsia on Hemorrhagic and Ischemic Stroke Risk: A 17 years Follow-up Nationwide Cohort Study
}

\author{
Chi-Jou Chuang ${ }^{1}$, Wen-Yen Chiou ${ }^{1}$, Hsuan-Ju Yang ${ }^{1}$, Shih-Kai Hung ${ }^{2}$, Moon-Sing Lee ${ }^{1}$, \\ Liang-Cheng Chen ${ }^{1}$, Chia-Hui Chew ${ }^{1}$, Ben-Hui $\mathrm{Yu}^{1}$, Feng-Chun Hsu ${ }^{1}$, and Hon-Yi Lin ${ }^{1}$ \\ ${ }^{1}$ Dalin Tzu Chi Hospital \\ ${ }^{2} \mathrm{Tzu}$ Chi University
}

December 3, 2021

\begin{abstract}
Objective No study ever investigated the long-term risk of stroke in women with pre-eclampsia/eclampsia. The purpose of this study is to explore long-term stroke risks, differentiating subtypes and their time trends. Design Nationwide population-based cohort study Methods Between 2000 and 2017, 1,384,427 pregnant women were registered in the National Health Insurance Research Database in Taiwan. After excluding women with previous stroke history and exact matching with all confounders, 6,053 women with pre-eclampsia/eclampsia and 24,212 controls were recruited. Main Outcome Measures Hemorrhagic and ischemic strokes after child-birth Results Over the 17-year follow-up, the adjusted hazard ratio (aHR) for stroke in women with a history of pre-eclampsia/eclampsia was 2.05 (95\% confidence interval, $\mathrm{CI}=1.67-2.52, \mathrm{p}<0.001)$. The 17 years overall risks of both ischemic and hemorrhagic stroke were 1.98 and 3.45 , respectively $(\mathrm{p}<0.001)$. The stroke subtypes, hemorrhagic and ischemic, had different time trend risks, and hemorrhagic stroke risks kept higher than that of ischemic stroke. The ischemic stroke risk peaked during 1-3 years after childbirth $(\mathrm{aHR}=3.09)$. The hemorrhagic stroke risk peaked during 3-5 years $(\mathrm{aHR}=7.49)$. Conclusions Stroke risk persisted even after decades, for both ischemic and hemorrhagic subtypes. Women with pre-eclampsia/eclampsia history should be aware of the long-term risk of stroke. Tweetable abstract Both ischemic and hemorrhagic stroke risks persisted high even after decades, while their time trend risks were different. Keywords: preeclampsia/eclampsia; ischemic stroke; hemorrhagic stroke
\end{abstract}

Impact of Pre-eclampsia/eclampsia on Hemorrhagic and Ischemic Stroke Risk: A 17 years Follow-up Nationwide Cohort Study

Authors:

Chi-Jou Chuang, MD ${ }^{\mathbf{a}, \mathbf{b}, \#}$, Wen-Yen Chiou, MD, PhD ${ }^{\mathbf{b}, \mathbf{c}}$, Hsuan-Ju Yang, MS ${ }^{\mathbf{c}, \# ~, ~ S h i h-K a i ~ H u n g, ~ M D, ~}$ $\mathrm{PhD}^{\mathbf{b}, \mathbf{c}}$, Moon-Sing Lee, MD, MS ${ }^{\mathbf{b}, \mathbf{c}}$, Liang-Cheng Chen, $\mathrm{MD}^{\mathbf{b}, \mathbf{c}}$, Chia-Hui Chew, $\mathrm{MD}^{\mathbf{c}}$, Ben-Hui Yu, $\mathrm{MS}^{\mathbf{c}}$, Feng-Chun Hsu, BS ${ }^{\mathbf{c}}$, Hon-Yi Lin, MD, PhD

$\mathbf{b}, \mathbf{c}, *$

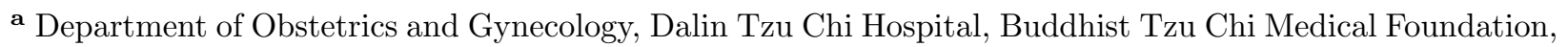
Chiayi, Taiwan

b School of Medicine, Tzu Chi University, Hualien, Taiwan

c Department of Radiation Oncology, Dalin Tzu Chi Hospital, Buddhist Tzu Chi Medical Foundation, Chiayi, Taiwan

\# These two authors contribute equally 
Corresponding author information: Hon-Yi Lin, MD, PhD

Hon-Yi Lin, MD, PhD

Department of Radiation Oncology, Dalin Tzu Chi Hospital, Buddhist Tzu Chi Medical Foundation, NO. 2, Min-Sheng Road. Dalin Town, Chiayi, Taiwan, R.O.C.

E-mail:doc31221@gmail.com;Telephone: +886 52648000 extension 5695

\section{Objective}

No study ever investigated the long-term risk of stroke in women with pre-eclampsia/eclampsia. The purpose of this study is to explore long-term stroke risks, differentiating subtypes and their time trends.

\section{Design}

Nationwide population-based cohort study

\section{Methods}

Between 2000 and 2017, 1,384,427 pregnant women were registered in the National Health Insurance Research Database in Taiwan. After excluding women with previous stroke history and exact matching with all confounders, 6,053 women with pre-eclampsia/eclampsia and 24,212 controls were recruited.

\section{Main Outcome Measures}

Hemorrhagic and ischemic strokes after child-birth

\section{Results}

Over the 17-year follow-up, the adjusted hazard ratio (aHR) for stroke in women with a history of preeclampsia/eclampsia was 2.05 (95\% confidence interval, $\mathrm{CI}=1.67-2.52, \mathrm{p}<0.001)$. The 17 years overall risks of both ischemic and hemorrhagic stroke were 1.98 and 3.45 , respectively $(\mathrm{p}<0.001)$. The stroke subtypes, hemorrhagic and ischemic, had different time trend risks, and hemorrhagic stroke risks kept higher than that of ischemic stroke. The ischemic stroke risk peaked during 1-3 years after childbirth $(\mathrm{aHR}=3.09)$. The hemorrhagic stroke risk peaked during $3-5$ years $(\mathrm{aHR}=7.49)$.

\section{Conclusions}

Stroke risk persisted even after decades, for both ischemic and hemorrhagic subtypes. Women with preeclampsia/eclampsia history should be aware of the long-term risk of stroke.

\section{Tweetable abstract}

Both ischemic and hemorrhagic stroke risks persisted high even after decades, while their time trend risks were different.

Keywords: pre-eclampsia/eclampsia; ischemic stroke; hemorrhagic stroke

\section{Introduction}

Pre-eclampsia/eclampsia, a hypertensive disorder of pregnancy, is a serious and common obstetric complication $^{1}$. The incidence of pre-eclampsia/eclampsia in pregnant women is $5-8 \%$ worldwide, and it is a major cause of maternal morbidity and mortality ${ }^{2-4}$. Pre-eclampsia/eclampsia is defined as hypertension with high levels of protein in their urine (i.e. proteinuria) after 20 weeks of gestation. Severe pre-eclampsia/eclampsia is a serious threat to the lives of mothers and fetuses, leading to cardiovascular and metabolic complications for mothers and persistent bradycardia, hypotension, and neonatal hypoglycemia for fetuses ${ }^{5-7}$. Pre-eclampsia/eclampsia may lead to serious kidney, brain, and blood clotting problems which cause $10-15 \%$ of maternal deaths in lower-income countries ${ }^{8,9}$.

Among these different sequelae of pre-eclampsia/eclampsia, stroke is a serious one. The incidence of stroke $^{10-13}$, both ischemic and hemorrhagic subtypes ${ }^{14,15}$, increased in the peripartum and postpartum stages. 
Stroke is an important issue not only in the elderly, but also in pregnant women. Stroke incidence ranges from 1.5 to 34.2 per 100,000 deliveries in pregnancy and the puerperium ${ }^{16,17}$. It has been reported that pre-eclampsia/eclampsia-related stroke would cause direct obstetric death ${ }^{18,19}$. The incidence of stroke in pregnant women in previous studies is varied according to different preexisting risk factors ${ }^{16,20}$.

It has been reported that the probability of cardiovascular ${ }^{7}$ and cerebrovascular disease ${ }^{10,21}$ in the next few years after childbirth in women with ever pre-eclampsia/eclampsia would to be higher than that in the general population. However, long-term impact of pre-eclampsia/eclampsia on cerebrovascular disease is unknown.

In this study, we aimed to utilize a nationwide database to explore the long-term overall, ischemic, and hemorrhagic stroke risks in women with a history of pre-eclampsia/eclampsia, after decades follow up.

\section{Materials and Methods}

This study was conducted following the Declaration of Helsinki. The study protocol was also approved by the Institutional Review Board (IRB) of Dalin Tzu Chi Hospital of Buddhist Tzu Chi Medical Foundation (approval number, B10402022). The IRB absolved the requirement for written informed consent due to no direct contact with individual patients from this de-identified database.

We used the Taiwan National Health Insurance Research Database (NHIRD) to analyze the incidence rate of stroke in women with pre-eclampsia/eclampsia and compared it to those without pre-eclampsia/eclampsia. Taiwan NHIRD contains all the records of diagnosis and treatment of approximately $99 \%$ of people from inpatient, outpatient, and emergency departments ${ }^{22}$. The data collection of pregnant women from the Taiwan NHIRD ranged from 2000 to 2017, and it was included in this study for statistical analysis. The data included were evaluated by the National Health Insurance Administration (NHIA) quarterly expert reviews on every 50 to 100 ambulatory and inpatient claims filed by each medical institution ${ }^{23}$. False diagnostic reports are subject to severe penalties from the NHIA ${ }^{24}$.

Records of pregnant women in this database were collected and categorized into two groups women, those with and without pre-eclampsia/eclampsia. Based on the International Classification of Disease, Ninth Revision, Clinical Modification (ICD-9-CM) codes for pregnant women, the codes were 650,651, 652, and 653 , while those for pregnant women with pre-eclampsia/eclampsia were $642.4,642.5,642.6$, and 642.7 . The primary outcomes included: codes for hemorrhagic stroke being 430,431, 432, and codes for ischemic stroke being 433, 434, 435, 436, and 437 .

Between 2001 and 2017, 1,384,427 pregnant women with delivery were registered in the Taiwan NHIRD. We included all pregnant women in Taiwan with different socioeconomic statuses, living areas (such as metropolises to rural), and hospital levels. The sample size exceeded million people. Figure 1 shows our study's flow diagram. We excluded 38,707 cases with missing confounders and 566 cases with stroke history. In addition, we only included women with the delivery age between 18 to 45 years. Finally, we enrolled 1,338,334 cases in this study, divided into groups of normal delivery with $(\mathrm{N}=8,077)$ and without $(\mathrm{N}=1,316,550)$ pre-eclampsia/eclampsia.

In this study, we used exact matching method to control covariates, that is, these two cohorts had the same age, same distribution for comorbidities, and socioeconomic status (all $p=1.000$ ). After 1:4 exact matching, 6,053 cases were selected in the pre-eclampsia/eclampsia group and 24,212 in the non-preeclampsia/eclampsia group. The follow-up time in this study was from 2000 to 2017. We divided the 17 years follow up into short (0-1, 1-3, 3-5 years), intermediate (5-10), and long (10-15 years), to further differentiate the risks at different intervals.

Covariates included were age, season, cesarean section or normal spontaneous delivery, multiple gestations, hospital levels, and comorbidities. The hospital level was included in the analysis, account for different care qualities during pregnancy at different hospital levels. The comorbidities included hypertension, gestational diabetes mellitus (GDM), anemia, and delivery conditions such as antepartum hemorrhage (APH) and postpartum hemorrhage (PPH). These young women, age 18 to 45, had no comorbidities, such as chronic 
kidney disease, heart failure, hypercholesterolemia, peripheral vascular disease, heart valve disorders, after matching. Socioeconomic variables, including geographic region, urbanization level, and monthly incomebased insurance premiums were analyzed to reduce bias resulting from lifestyle.

Statistical methods

The basic characteristics between the two study groups were compared by Chi-square test. We used a Cox regression model to compare the adjusted hazard ratio (aHR) and 95\% confidence interval (CI) for stroke occurrence. Cumulative incidence functions for the first occurrence of stroke episode between pre-eclampsia/eclampsia and non-pre-eclampsia/eclampsia women were compared using the Kaplan-Meier model. The SAS software (version 9.2; SAS Institute, Inc., Cary, NC) was used for all statistical analyses. A two-sided $P$-value of $<0.05$ was considered statistically significant.

\section{Results}

Demographic characteristics of pre-eclampsia/eclampsia and non-pre-eclampsia/eclampsia cohorts

The mean ages of the groups with and without pre-eclampsia/eclampsia were 32.04 and 31.84 years, respectively. No statistically differences in distribution of the all demographic characteristics and comorbidities between two groups after exact matching.

Prevalence rates of stroke in these matched cohorts

During this 17-year follow up time, 2.15\% (130 over 6,053) women in the pre-eclampsia/eclampsia group, ever had stroke which was higher than that in the non-pre-eclampsia/eclampsia group $(1.23 \%, \mathrm{p}<0.001)$ (Table 1). The prevalence rates of ischemic and hemorrhagic stroke in the pre-eclampsia/eclampsia group were also higher than that in the non-pre-eclampsia/eclampsia group ( $1.88 \%$ versus $1.12 \%, 0.55 \%$ versus $0.18 \%$, respectively, both $\mathrm{p}<0.001)$.

Risks of stroke

Table 2 presents the aHRs of stroke after adjusting for covariates. Stroke risk was significantly higher in the pre-eclampsia/eclampsia group than in the non-pre-eclampsia/eclampsia group (aHR $=2.05,95 \% \mathrm{CI}$ $=1.67-2.52, p<0.001)$.

Women with old delivery age had higher risk of stroke than that of women with young delivery age (Table $2)$, with highest risk being $2.61(p<0.001)$ for age $>35$ years, compared to women with age at delivery $<30$ years.

From the Cox regression analysis results presented in Table 3, delivery-related variables, such as cesarean section, multiple gestation, hospital level, and the season of maternal delivery did not have significant risk for stroke.

For comorbidities, Hypertension increased risks for stroke with aHR $3.35(95 \% \mathrm{CI}=1.99-5.63, p<0.001)$ in these two matched cohorts. APH had a significant risk for stroke. Other comorbidities such as GDM, anemia, and PPH had no significant impact on stroke occurrence.

Socioeconomic variables, such as geographic region and urbanization level, had no significant stroke risk except family income. Women in median or high-income families had a higher risk of stroke than that of women in low-income families.

Risks of stroke for different stroke subtypes

Table 3 presents the aHRs of ischemic stroke, and hemorrhagic stroke, after adjusting for covariates. The aHR of ischemic stroke was $1.98(95 \% \mathrm{CI}=1.59-2.46, p<0.001)$ and the aHR of hemorrhagic stroke 3.45 $(95 \% \mathrm{CI}=2.18-5.47, p<0.001)$, respectively, for overall 17 years follow-up.

Risks of stroke for different follow-up time interval 
We also observed stroke risk in women with pre-eclampsia/eclampsia at intervals of 0-1, 1-3, 3-5, 5-10, and 10-15 years (see Table 3). Overall, the stroke risk reach peak within 1-3 years after child delivery (aHR 3.20, $95 \% \mathrm{CI}=1.82-5.63, p<0.001$ ). The risk time trends for these two stroke subtypes were different (Table 3 and as illustrated in Figure 2). The ischemia stroke risk reach peak within 1-3 after child delivery (aHR 3.09, $95 \% \mathrm{CI}=1.71-5.58, p<0.001$ ), while hemorrhage stroke reach peak within $3-5$ years after child delivery (aHR $7.49,95 \% \mathrm{CI}=1.18-47.33, p=0.032$ ). Besides, the hemorrhagic stroke risk was higher than the ischemic risk at each follow-up time interval.

Cumulative incidence rates of stroke

The 17 years cumulative incidence rates of overall stroke, ischemic and hemorrhagic stroke were all higher in women with pre-eclampsia/eclampsia history than that in women without pre-eclampsia/eclampsia $(6.00 \%$ versus $3.01 \%, 5.05 \%$ versus $2.62 \%, 1.95 \%$ versus $0.57 \%$, all $p<0.001$ ) as Figure 3.

\section{Discussion}

\section{Main Findings}

Overall, women with pre-eclampsia/eclampsia in pregnancy significantly increased the future occurrence rate of stroke, both ischemic and hemorrhagic stroke, even after 10 to 15 years. The risks of hemorrhagic stroke at different follow up periods were all higher than that of ischemic stroke, with different risk time trends. The peak of ischemic stroke risk was 1-3 years after childbirth; while for the peak of hemorrhagic stroke risk was 3-5 years after childbirth.

Strengths and Limitations

First, this study's major strength is exploring the association between pre-eclampsia/eclampsia and stroke in a nationwide scope cohort study. Second, this study reported the two different stroke subtypes and divided the long follow-up duration into different follow-up durations to provide information regarding the timetrend effects of pre-eclampsia/eclampsia on stroke. Finally, because of the large number of women in this nationwide study, we used exact matching to control all covariates, that is, women with the same age, same comorbidity distributions, and same socioeconomic status, which could reduce statistical bias in this study.

This study has several limitations. First, our health insurance database provides no information on laboratory data, nor the severity of pre-eclampsia/eclampsia. Second, smoking habits, alcohol consumption, or body weight/body mass index are not available in our database. Therefore, we could not evaluate the effects of these factors on stroke.

\section{Interpretation}

Our results show that a history of pre-eclampsia/eclampsia in pregnancy significantly increased the future occurrence rate of stroke, both ischemic and hemorrhagic stroke, even after 10 to 15 years. In this study, after adjusting for potential confounding variables, women with history of pre-eclampsia/eclampsia still had a 2-fold higher long-term stroke risk. Nearly 2-fold higher ischemic stroke risk and up to 3-fold higher hemorrhagic stroke risk were observed.

Reviewing the literature, it has been often reported that pre-eclampsia/eclampsia would increase stroke risk during the pregnancy process, both ischemic and hemorrhagic stroke, a dangerous complication of pregnancy ${ }^{11,25-30}$. In those studies, pre-eclampsia/eclampsia generally increased a woman's stroke risk and hypertension between $2^{\sim} 4$-fold during pregnancy, respectively ${ }^{14,30-33}$, and also leads to higher mortality of pregnancy and delivery $2,34,35$. In a case-control stroke study, Kittner and colleagues found that the adjusted relative risk (aRR) of stroke (both cerebral infarction and intracerebral hemorrhage) during pregnancy and the 6-week postpartum period was 2.4 (95\% CI, 1.6 to 3.6$)^{36}$. The aRR during pregnancy was $1.1(95 \%$ CI, 0.6 to 2.0), while for risk during the 6-week postpartum period alone the aRR increased to 7.9 (95\% CI, 5.0-12.7). Compared to our cohort study, our study showed aHR was 1.65 during $0-1$ years after childbirth, 3.20 during $1-3$ years, and 1.80 during $10-15$ years. 
In the literature, another case-control study with follow-up time prolonging to one year after childbirth, Tang et al. evaluated the aRRs of ischemic and hemorrhagic stroke during pregnancy and the first postpartum year ${ }^{14}$. In that study, they found a U-shape trend of hemorrhagic stroke risk from antepartum to 1-year postpartum (aRR 10.68, to 6.45, to 5.61, to 11.76, to 19.90 for 3 months antepartum, and 3 days, 6 weeks, 6 months, 12 months postpartum, respectively). Compared to that U shape of that short postpartum casecontrol study, our long-term cohort study showed a reverse-U shape in decade follow-up for hemorrhagic stroke with a peak of aHR 7.49 during 3-5 years after childbirth. .

For the ischemic stroke risk, Tang's study showed aRR as high as 40.86 within 3 months antepartum, then decrease to 11.23 from 3 days to 6 weeks postpartum, and further decreased to 4.35 from 6 months to 12 months postpartum. In our cohort study, the aHR for ischemic stroke increased from 1.82 during the first year after childbirth, with peak 3.09 during 1-3 years, and then decreased to 1.58 after 10 years.

In the literature, it has been reported that ischemic stroke had two peaks of occurrence, in the first and third trimesters of pregnancy: 24/27 (89\%), while intracerebral hemorrhage was more frequently observed during the third trimester: $15 / 23(65 \%)^{37}$. Different from previous studies, in our long-term follow-up study, we noted that stroke risk would reach peak during 1-3 years after childbirth for ischemic stroke and during 3-5 years for hemorrhagic stroke. Based on short-term follow-up studies in the past and this long-term study, it is found consistently that ischemic stroke occurs faster and earlier than hemorrhagic stroke.

The role of hypertension in women with pre-eclampsia/eclampsia is also worth to note in this study. In previous studies, compared to patients without pre-existing hypertension those with higher blood pressure have smaller amount of salvageable tissue and obvious intracranial occlusion and thus worsen stroke outcome $^{38-41}$. Both of the elevation of systolic and diastolic blood pressure increase stroke risk of 2.9 -fold for women $^{42,43}$. In addition, recent study further identifies related genes of predisposing to hypertension may associate with pre-eclampsia/eclampsia in Asian women ${ }^{44}$. Our study confirm the role of hypertension on stroke in women with history of pre-eclampsia/eclampsia with aHR 3.35 (95\% CI 1.99-5.63). For women with pre-eclampsia/eclampsia, the role of hypertension is a red flag in aggravating probability of future stroke occurrence.

Age may also have significant influence on stroke. Previous studies ever discussed the impact of maternal age on stroke risk ${ }^{13,45,46}$. For example, women aged $>39$ years and those aged $>29$ years are significant hemorrhagic and ischemic stroke factors associated with increased risk, respectively ${ }^{46}$. This is consistent with our results, which showed women with an age at delivery $>35$ years had a significantly high aHR of hemorrhagic stroke risk.

For socioeconomic status, previous studies suggest that socioeconomic deficiency is associated with increased stroke severity and incidence at young age population ${ }^{47}$, and stroke mortality ${ }^{47,48}$. This influence of socioeconomic deficiency may have a greater impact on pregnant women, which may cause pregnant women to have a higher stroke risk than other groups ${ }^{49}$. Our long-term study also showed that women with lowest family income had elevated stroke risk than women with other family income levels.

\section{Conclusions}

In summary, our results indicate that the ischemic and hemorrhagic stroke risk of women with preeclampsia/eclampsia history remained high for a long time. Women with pre-eclampsia/eclampsia history should be aware of stroke risk, even 10 to 15 years after childbirth.

\section{Disclosure of interests}

All authors declare that they have no competing interests.

\section{Acknowledgements}

HYL, SKH, WYC, MSL, and LCC acquired the data and supervised the project. CJC, SKH, HYL, and WYC conceived and designed the study, with input from the other authors. BHY, HJY, and FCH performed 
the statistical analyses. WYC and HJY wrote the first draft of the manuscript. All authors interpreted the data and contributed to the writing of the paper. All authors revised and approved the final version.

\section{Details of Ethics approval}

The present study was conducted and approved after a formal approval of Institute Review Board in Dalin Tzu Chi Hospital, Buddhist Tzu Chi Medication Foundation (approval number, B10402022). We confirm that this report is consistent with ethical publication.

\section{Funding}

We appreciate the research grants from the Dalin Tzu Chi Hospital (grant number: DTCRD106-I-09). The funders had no role in the study design, data collection and analysis, decision to publish, or the preparation of the manuscript.

\section{References}

1. National High Blood Pressure Education Program Working Group Report on High Blood Pressure in Pregnancy. American journal of obstetrics and gynecology. Nov 1990;163(5 Pt 1):1691-1712.

2. Ghulmiyyah L, Sibai B. Maternal mortality from preeclampsia/eclampsia. Seminars in perinatology. Feb 2012;36(1):56-59.

3. AnanthKarumanchi SEM.Molecular and Genetic Basis of Renal Disease. Elsevier; 2008.

4. Duley L. The global impact of pre-eclampsia and eclampsia. Seminars in perinatology. Jun 2009;33(3):130-137.

5. Heida KY, Zeeman GG, Van Veen TR, Hulzebos CV. Neonatal side effects of maternal labetalol treatment in severe preeclampsia. Early human development. Jul 2012;88(7):503-507.

6. Hermes W, Franx A, van Pampus MG, et al. Cardiovascular risk factors in women who had hypertensive disorders late in pregnancy: a cohort study. American journal of obstetrics and gynecology. Jun 2013;208(6):474 e471-478.

7. Mongraw-Chaffin ML, Cirillo PM, Cohn BA. Preeclampsia and cardiovascular disease death: prospective evidence from the child health and development studies cohort. Hypertension (Dallas, Tex. : 1979). Jul 2010;56(1):166-171.

8. Duley L. Maternal mortality associated with hypertensive disorders of pregnancy in Africa, Asia, Latin America and the Caribbean. British journal of obstetrics and gynaecology. Jul 1992;99(7):547-553.

9. Khan KS, Wojdyla D, Say L, Gülmezoglu AM, Van Look PF. WHO analysis of causes of maternal death: a systematic review. Lancet (London, England). Apr 1 2006;367(9516):1066-1074.

10. Park Y, Cho GJ, Kim LY, Lee TS, Oh MJ, Kim YH. Preeclampsia Increases the Incidence of Postpartum Cerebrovascular Disease in Korean Population. Journal of Korean medical science. Feb 5 2018;33(6):e35.

11. Sells CM, Feske SK. Stroke in Pregnancy. Seminars in neurology. Dec 2017;37(6):669-678.

12. Wu P, Haththotuwa R, Kwok CS, et al. Preeclampsia and Future Cardiovascular Health: A Systematic Review and Meta-Analysis. Circulation. Cardiovascular quality and outcomes. Feb 2017;10(2).

13. Hovsepian DA, Sriram N, Kamel H, Fink ME, Navi BB. Acute cerebrovascular disease occurring after hospital discharge for labor and delivery. Stroke. Jul 2014;45(7):1947-1950.

14. Tang $\mathrm{CH}, \mathrm{Wu} \mathrm{CS}$, Lee TH, et al. Preeclampsia-eclampsia and the risk of stroke among peripartum in Taiwan. Stroke. Apr 2009;40(4):1162-1168.

15. Brown DW, Dueker N, Jamieson DJ, et al. Preeclampsia and the risk of ischemic stroke among young women: results from the Stroke Prevention in Young Women Study.Stroke. Apr 2006;37(4):1055-1059. 
16. Tate J, Bushnell C. Pregnancy and stroke risk in women. Women's health (London, England). May 2011;7(3):363-374.

17. James AH, Bushnell CD, Jamison MG, Myers ER. Incidence and risk factors for stroke in pregnancy and the puerperium. Obstetrics and gynecology. Sep 2005;106(3):509-516.

18. MacKay AP, Berg CJ, Atrash HK. Pregnancy-related mortality from preeclampsia and eclampsia.Obstetrics and gynecology. Apr 2001;97(4):533-538.

19. Ananth CV, Savitz DA, Bowes Jr. WA. Hypertensive disorders of pregnancy and stillbirth in North Carolina, 1988 to 1991. Acta Obstetricia et Gynecologica Scandinavica. 1995;74(10):788-793.

20. Moatti Z, Gupta M, Yadava R, Thamban S. A review of stroke and pregnancy: incidence, management and prevention. European journal of obstetrics, gynecology, and reproductive biology. Oct 2014;181:20-27.

21. van Veen TR, Panerai RB, Haeri S, Griffioen AC, Zeeman GG, Belfort MA. Cerebral autoregulation in normal pregnancy and preeclampsia. Obstetrics and gynecology. Nov 2013;122(5):1064-1069.

22. Lu JF, Hsiao WC. Does universal health insurance make health care unaffordable? Lessons from Taiwan. Health affairs (Project Hope). May-Jun 2003;22(3):77-88.

23. Cheng TM. Taiwan's new national health insurance program: genesis and experience so far. Health affairs. May-Jun 2003;22(3):61-76.

24. Website BoNHI. Methods for estimating false claims. 2000.

25. Miller EC, Gatollari HJ, Too G, et al. Risk Factors for Pregnancy-Associated Stroke in Women With Preeclampsia. Stroke. Jul 2017;48(7):1752-1759.

26. Sidorov EV, Feng W, Caplan LR. Stroke in pregnant and postpartum women. Expert review of cardiovascular therapy. Sep 2011;9(9):1235-1247.

27. Fairhall JM, Stoodley MA. Intracranial haemorrhage in pregnancy. Obstetric medicine.Dec 2009;2(4):142148.

28. Vilela P, Duarte J, Goulao A. [Cerebrovascular disease in pregnancy and puerperium].Acta medica portuguesa. Jan-Feb 2001;14(1):49-54.

29. Pankiewicz K, Szczerba E, Maciejewski T, Fijalkowska A. Non-obstetric complications in preeclampsia. Przeglad menopauzalny = Menopause review. Jun 2019;18(2):99-109.

30. Bushnell C, McCullough LD, Awad IA, et al. Guidelines for the prevention of stroke in women: a statement for healthcare professionals from the American Heart Association/American Stroke Association. Stroke. May 2014;45(5):1545-1588.

31. Miller EC, Leffert L. Stroke in Pregnancy: A Focused Update. Anesthesia and analgesia.May 202019.

32. Sanders BD, Davis MG, Holley SL, Phillippi JC. Pregnancy-Associated Stroke. Journal of midwifery $E^{3}$ women's health. Jan 2018;63(1):23-32.

33. Razmara A, Bakhadirov K, Batra A, Feske SK. Cerebrovascular complications of pregnancy and the postpartum period. Current cardiology reports. 2014;16(10):532.

34. Tranquilli AL. Prediction, medical illness and the risk of pre-eclampsia.Pregnancy hypertension. Jul $2014 ; 4(3): 245$.

35. Wilson BJ, Watson MS, Prescott GJ, et al. Hypertensive diseases of pregnancy and risk of hypertension and stroke in later life: results from cohort study.BMJ (Clinical research ed.). Apr 19 2003;326(7394):845.

36. Kittner SJ, Stern BJ, Feeser BR, et al. Pregnancy and the risk of stroke. The New England journal of medicine. Sep 12 1996;335(11):768-774. 
37. Cantu-Brito C, Arauz A, Aburto Y, Barinagarrementeria F, Ruiz-Sandoval JL, Baizabal-Carvallo JF. Cerebrovascular complications during pregnancy and postpartum: clinical and prognosis observations in 240 Hispanic women. European journal of neurology. Jun 2011;18(6):819-825.

38. Cipolla MJ, Liebeskind DS, Chan SL. The importance of comorbidities in ischemic stroke: Impact of hypertension on the cerebral circulation. Journal of cerebral blood flow and metabolism : official journal of the International Society of Cerebral Blood Flow and Metabolism. Dec 2018;38(12):2129-2149.

39. Lima FO, Furie KL, Silva GS, et al. The pattern of leptomeningeal collaterals on CT angiography is a strong predictor of long-term functional outcome in stroke patients with large vessel intracranial occlusion. Stroke. Oct 2010;41(10):2316-2322.

40. Ahmed N, Wahlgren N, Brainin M, et al. Relationship of blood pressure, antihypertensive therapy, and outcome in ischemic stroke treated with intravenous thrombolysis: retrospective analysis from Safe Implementation of Thrombolysis in Stroke-International Stroke Thrombolysis Register (SITS-ISTR). Stroke. Jul 2009;40(7):2442-2449.

41. Leonardi-Bee J, Bath PM, Phillips SJ, Sandercock PA. Blood pressure and clinical outcomes in the International Stroke Trial. Stroke. May 2002;33(5):1315-1320.

42. Kannel WB, Wolf PA, Verter J, McNamara PM. Epidemiologic assessment of the role of blood pressure in stroke. The Framingham study. Jama. Oct 12 1970;214(2):301-310.

43. Kannel WB, Wolf PA, McGee DL, Dawber TR, McNamara P, Castelli WP. Systolic blood pressure, arterial rigidity, and risk of stroke. The Framingham study.Jama. Mar 27 1981;245(12):1225-1229.

44. Steinthorsdottir V, McGinnis R, Williams NO, et al. Genetic predisposition to hypertension is associated with preeclampsia in European and Central Asian women. Nature communications. Nov 25 2020;11(1):5976.

45. Leffert LR, Clancy CR, Bateman BT, Bryant AS, Kuklina EV. Hypertensive disorders and pregnancyrelated stroke: frequency, trends, risk factors, and outcomes. Obstetrics and gynecology. Jan 2015;125(1):124131.

46. Too G, Wen T, Boehme AK, et al. Timing and Risk Factors of Postpartum Stroke. Obstetrics and gynecology. Jan 2018;131(1):70-78.

47. Aslanyan S, Weir CJ, Lees KR, Reid JL, McInnes GT. Effect of area-based deprivation on the severity, subtype, and outcome of ischemic stroke. Stroke. Nov 2003;34(11):2623-2628.

48. Wu SH, Woo J, Zhang XH. Worldwide socioeconomic status and stroke mortality: an ecological study. International journal for equity in health. Jun 15 2013;12:42.

49. Kim MK, Lee SM, Bae SH, et al. Socioeconomic status can affect pregnancy outcomes and complications, even with a universal healthcare system. International journal for equity in health. Jan 5 2018;17(1):2.

\section{Figure legends}

Figure 1. A flow chart illustrating the selection procedure of study subjects. PE; pre-eclampsia/eclampsia.

Figure 2. The risk time trends of ischemic and hemorrhagic stroke in women with a history of preeclampsia/eclampsia.

Figure 3. Cumulative event rates of overall strokes (A), ischemic stroke (B), and hemorrhagic stroke (C) among women with and without history of pre-eclampsia/eclampsia. 

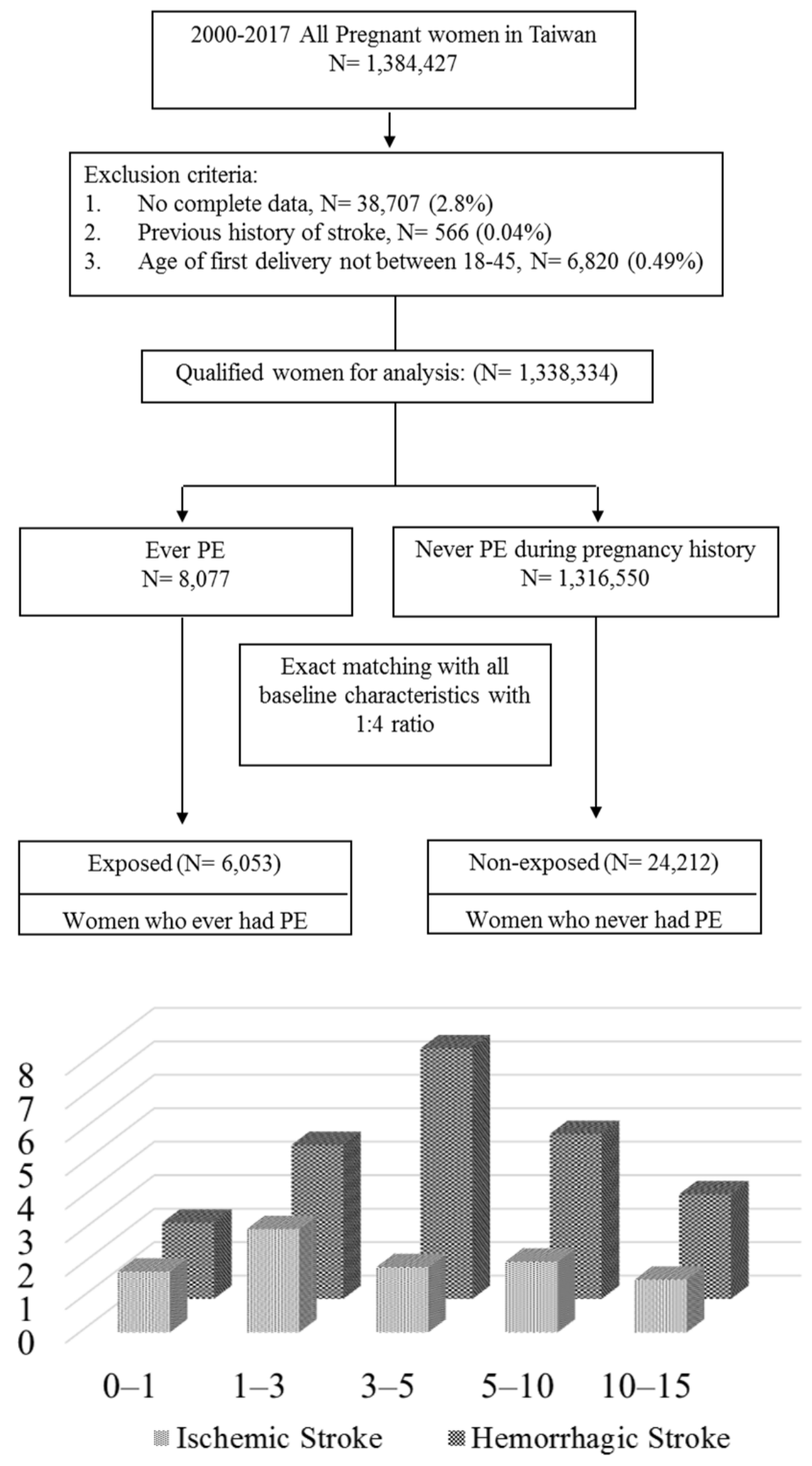
A

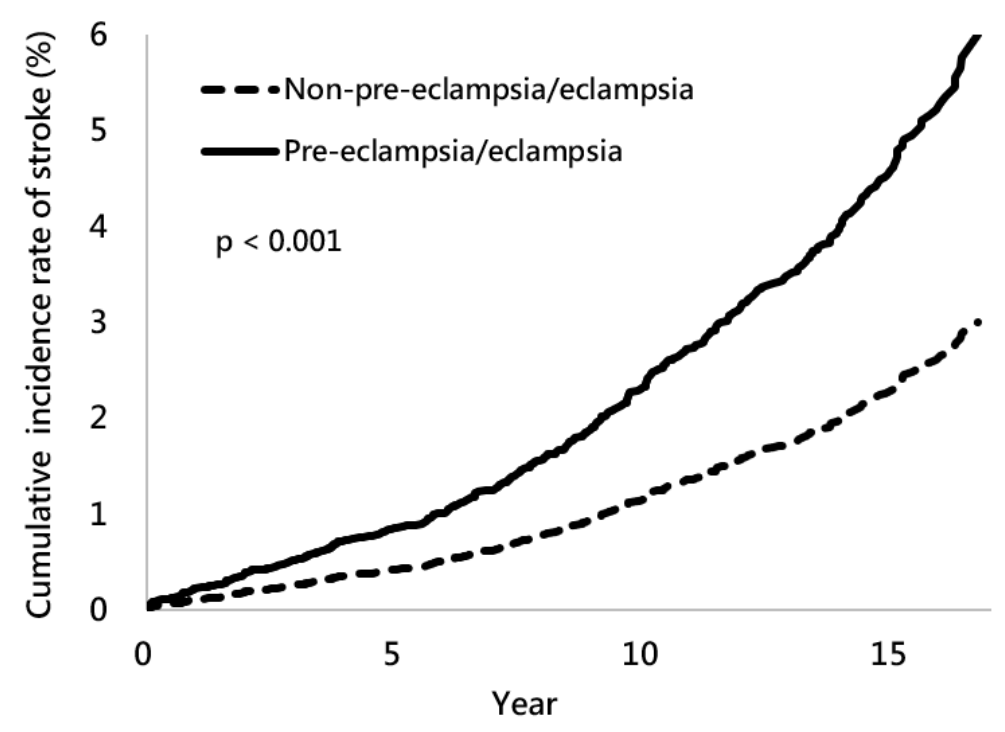

B

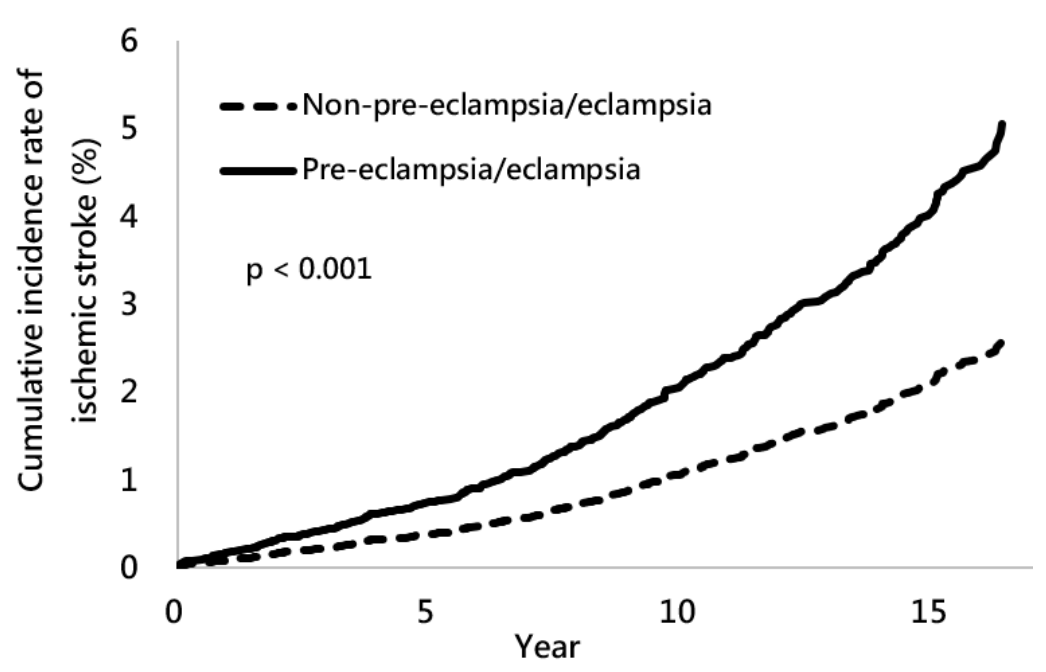

C

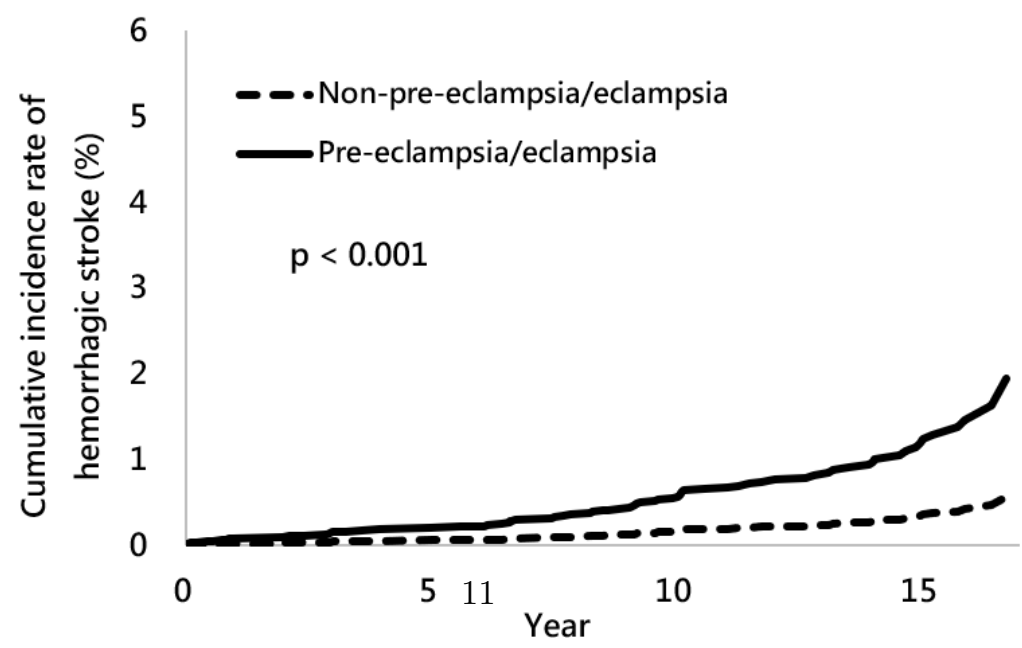




\section{Hosted file}

(+)Table 1_preeclampsia.docx available at https://authorea.com/users/449371/articles/547916impact-of-pre-eclampsia-eclampsia-on-hemorrhagic-and-ischemic-stroke-risk-a-17-yearsfollow-up-nationwide-cohort-study

\section{Hosted file}

(+)Table 2_preeclampsia.docx available at https://authorea.com/users/449371/articles/547916impact-of-pre-eclampsia-eclampsia-on-hemorrhagic-and-ischemic-stroke-risk-a-17-yearsfollow-up-nationwide-cohort-study

\section{Hosted file}

(+)Table 3_preeclampsia.docx available at https://authorea.com/users/449371/articles/547916impact-of-pre-eclampsia-eclampsia-on-hemorrhagic-and-ischemic-stroke-risk-a-17-yearsfollow-up-nationwide-cohort-study 